\title{
A Data Centric approach for Workflows
}

\author{
A. Akram, J Kewley and R. Allan \\ CCLRC e-Science Centre, Daresbury Laboratory, Warrington, UK WA4 4AD \\ \{a.akram, j.kewley, r.j.allan\}@dl.ac.uk
}

\begin{abstract}
The complexity, unpredictability and inter-dependency of the components in a workflow often demand thorough planning at the design level. In this paper we propose a Data-Centric approach to instantiate, monitor and manage complex workflows. We believe a culture change in the orchestration of services is required to consider their data dependency while modelling workflows. The current process or activity oriented mechanism, utilized in the existing workflow technologies, failed to live up to expectations mainly due to data issues at later stages.
\end{abstract}

\section{Introduction}

Grid computing has been identified as an important new technology by a wide range of scientific and engineering fields and by many commercial and industrial enterprises [1]. Grid computing tried to address the challenge of integrating services, spread across distributed, heterogeneous, dynamic Virtual Organizations using the concepts and technologies from the Web Services community. These Web Services can reach their full potential only if there exists a mechanism to describe the various interactions between the services and dynamically compose new services out of existing ones. Existing technologies, addressing the workflow for the Web Services are based on the Service Oriented Architecture (SOA). An SOA is an architectural style whose goal is to achieve loose coupling among interacting software agents (services and clients). These services can communicate with each other, either through explicit messages (which are descriptive rather than instructive), or by a number of 'master' services that coordinate or aggregate activities together, typically in a workflow.

In recent years, Web Services have been established as a popular "connection technology" for implementing an SOA. Web Services are defined as a platform and implementation independent software component that can be [2]:

- Described using a service description language
- Published to a registry of services

- Discovered through standard mechanisms

- Invoked through a declared API

- Composed with other services

The well-defined interface required for services is described in a WSDL file (Web Service Description Language [3]). Services exposed as Web Services can be integrated into complex workflows which may span multiple domains and organizations.

A workflow is defined as the automation of procedures where documents, information, or tasks are passed between participants according to a defined set of rules to achieve, or contribute to, an overall business goal. We believe that culture change in workflow orchestration is essential with stronger consideration being given to data dependencies of the participating services during design. This suggested change is based on the Data-Centric Model (Section 2.1) which compliments the existing Service Oriented Model (Section 2.2). In the Data Centric model workflow is orchestrated around Data Type and Data Flow rather than the nature and the capabilities of the individual services only.

The Web Services Resource Framework (WS-RF) [5] specification built on top of existing Web Service standards addresses the limitation of stateless Web Services through the concept of WS-Resources (Section 3.1). WS-RF defines the conventions within the context of established Web Services standards for managing a state so that applications can reliably share changing information, and discover, inspect and interact with stateful resources in a standard and interoperable way [6]. WS-RF specifications can enhance the Data-Centric workflow, without extending or rewriting existing specifications (Section 4).

\section{Workflow}

According to Kammer [7] "The unpredictability of the business processes requires workflow systems supporting the exception handling with the ability to dynamically adapt to the changing environment". 
Traditional approaches to handle this problem have fallen short, providing little support for the change, particularly once the process has begun execution. Dynamic workflows often need to be complemented with execution support at runtime, such as dynamic scheduling, dynamic resource (i.e. data) binding, and infrastructure reconfiguration. These constraints demand flexibility in a workflow language. Traditionally, workflows were solely designed to call different Web Services in a static manner based on their operation semantics only.

\subsection{Data-Centric Workflow}

Our approach for designing a workflow is based on the modified Data-Flow Diagrams (DFDs), which we call as Data Centric Workflow. DFDs show the flow of data from external entities into the system, how the data moved from one process to another, as well as its logical storage. Workflow scripts in the context of Web Services only focus on the nature of the data flowing from one process to another rather than how it is moved.

The process of identifying, modelling and documenting what data moves around an information system is called Data Flow Modelling. Data flow modelling examines processes (activities that transform data from one form to another), data stores (the holding areas for data), external entities (what sends data into a system or receives data from a system), and data flows (routes by which data can flow). The purpose of data modelling is to develop an accurate model, of the different business requirements, stake holders, and sub-processes and activities.

In the data-centric workflow, the first step is to define data definitions in the context of the application. The initial goal in a data-centric workflow is not only to visualize the initial or final data set involved in the whole process but also to capture the intermediate data sets used by different sub-processes during the lifecycle of the application. At the workflow design stage, more emphasis should be placed on the possible transformations of the data, routed between different interacting activities and processes. The services (i.e. Web Services) offered by different business partners, collaborators or parties forming the supply chain follow the Data Flow Model. In this approach, Web Services are developed in accordance with already negotiated and accepted data models. The role of any such Web Service is pre-defined in the context of an application, in the form of its responsibility, data input and output, and expected functionalities. Web Services from multiple vendors, partners and collaborators may fit in the same role and hence they can be replaceable in the overall integration process or workflow.

This means current services may need to be reengineered to share common data model and provide predictable services. These services are developed in accordance with the application data model and are less likely to change on an ad hoc basis. The services may also define their own inner data structure but then these services need to develop their mapping routines and interfaces in compliance with the Data Model. The reusability of the services (tightly coupled with any specific data model) can be limited across multiple domains with different applications but may have overlapping data models. The reusability limitation of services can be an issue only for those enterprise applications which have a strictly defined data model. The service can improve its effectiveness by declaring multiple inner data mapping routines to comply with different overlapping data models.

The practical solution for any large enterprise to integrate business processes is to consolidate its operational data to speed up the data flow across tasks. With a consolidated and structured data model shared by different activities or business process (i.e. order entry, fulfilment and distribution applications), the enterprise no longer suffers issues related to the data redundancy, operational disruptions caused by data errors and enjoys improved process execution and customer services.

Today's Holy Grail is metadata - that is, a common source of definitions and context about the data. Metadata plays an important role in data management to establish a higher level of shared information resources. Often, each application has its own metadata, which must be coordinated through a shared data vocabulary. In the shared data vocabulary, data has common definitions, names and integrity rules.

In enterprise organizations the business relationships and activity co-ordinations are becoming complex and they often demand simplicity in the integration of their services. Business users want common interfaces, business processes, application functionality, tools and services. These simplifications on different fronts can only be achieved by adhering to specific constraints, and the pre-defined data model is an appropriate approach. A data model provides the single vision of its application domain, no matter how many different stakeholders with their individual perspectives are involved.

Businesses need the technologies to work together to solve integration problems, but currently most of the developing technologies increase the integration problems by adding technology related complexities. 
The data-centric approach can solve the integration problem in different possible aspects outlined below:

2.1.1. Reusable Data Set. Modeling the data without concerning implementation details assures that same data can be used in different services. In the Web Services context, services only provide interface details (i.e. operations and the service endpoint locations) in the WSDL by importing externally declared schemas. The reusable data set minimize the issues related to overlapping data sets and different XML Namespaces at the integration level. Existing Schemas can be reused rather than re-designing a new data type system for each new Web Service. This helps reduce development efforts, cost and time.

2.1.2. Agreed Data Model. All stakeholders may negotiate before agreeing the data model, which means they form the contract to provide the service interfaces according to the agreed data model. This encourages co-operation among the various stakeholders from the early stages of the enterprise application design.

2.1.3. Shared Data Vocabulary. Different stakeholders and their offered services share the same data definition, meaning and relationship of the data. They should also have a similar view and structure of the data, common integrity rules and data constraints. This establishes an agreed data vocabulary which provides consistency while integrating geographical dispersed services.

2.1.4. Standard compliant Data Models. The data itself is described in XML and the data model is designed using XML Schemas. The XML Schema based data leverages the more powerful features of the XML language for description, constraint and validation of complex data (e.g., XSD patterns, regular expressions, optional elements, enumerations and type restrictions). In practice, this has proved invaluable for the description and constraint of complex enterprise and scientific data.

2.1.5. Uniform View. The performance management and collaborative business relationships demand a single view of the relevant standard-compliant information. Each service takes responsibility and plays its role in the integrated application, knowing what is expected of it and what it can expect from other services. These individual services can even invoke each other directly without involvement of the workflow engine.

2.1.6. Simple Integration. The business logic of different Web Services is based on the same data model, without requiring any sort of data transformation and data mapping at the integration level. Any data mapping required is provided internally by the service to comply with the agreed data model.
2.1.7. Single Source of Modification. Declaration of the data external to the participating Web Services provides a single source of information, updates and modifications. This isolation of data therefore limits its impact on other Web Service components such as the concrete WSDL file implementation. All the modifications and update to the data should be with the consent of the participating stake holders.

2.1.8. Improved Performance. Research has shown that distributed data-flow models can offer better performance and lower communication costs in its service composition than the centralized models. However, an impediment towards Data-flow distribution in Web services composition is that the component services cannot exchange data directly without central mediation [8].

2.1.9. Separation of Roles. The Data Type system can be fully abstracted and developed in isolation from the network protocol and communication specific details of the WSDL file. In doing this, the focus becomes centred upon the business/scientific requirements of the data model. In our experience, this greatly encourages collaboration, particularly between the scientists who are involved with the description of scientific data and data model design.

2.1.10. Data Binding and Validation. Most of the current SOAP Engines provide minimum data binding and validation. This lack of data validation may result in non-compliant message exchange between services. This results in run time errors beyond immediate control of the developer. Services can make use of external data binding and validation tools which are $100 \%$ compliant with XML Schemas for the construction and validation of Web Service messages and instance documents.

2.1.11. Stability. In the data-centric approach, different partner services involved in the workflow are bound to two contracts: the WSDL contract and the data model contract. The workflow engine has no control on the WSDLs related to the partner services but can control their behaviour through the data model. This double contract limits the ad hoc changes in the constituting services and results in a more stable workflow.

Due to the stateless nature of the vanilla Web Services, it is quite difficult to develop data-centric workflows if not impossible. The lack of state notion in the Web Services Architecture [15] restricts any automatic integration of the services without customization. Such home-grown extensions for the Web Services Architecture could kill its basic purpose of interoperability and platform and framework independence. The Web Services Resource Framework (WS-RF) provides a standard way to model the state of 
Web Services which can be shared among partner services. In the WS-RF specifications state is modelled as WS-Resource. WS-Resources are the dynamic representation of the 'state' and can be updated or modified at the run time. We have proposed the solution for Data-Centric Workflows based on the existing standards of the WS-RF specification. In the section 4, we describe our vision of modelling states in Web Services and how this concept of states can map to the data model of any enterprise application (EA).

\subsection{Service Oriented Workflow}

In recent years, Web Services have been established as a popular "connection technology" for implementing a SOA. With the popularity of Web Services, several composition languages for Web Services have emerged in the last few years, such as BPML, XLANG [10], WSFL [9], WSCDL [12], BPEL4WS [11], GSFL [13] and WS Orchestration. Most of these composition languages address the orchestration problem by interrelating different services and activities in the control flow. The normal approach practiced in existing workflow languages is to construct the workflow script based on the available Web Services and their operations. The Web Services are autonomous processes which can work independently from specific data dependency. The workflow script orchestrating these autonomous services requires additional work to resolve data conflicts between different services. The normal steps involved in producing any complicated workflow can be summarized as:

1. Discovering suitable Web Services;

2. Parsing WSDLs;

3. Extracting the Data Information from WSDLs;

4. Data Mapping to match the service requirements;

5. Data Transformation at each activity level;

6. Resolving the Namespace issues related to different data sets used by individual services.

Apparently these steps seem very systematic, logical and simple. Our experiences of orchestrating complex workflows, with the various stakeholders involved, have exposed different data specific issues. Most of the efforts spent in this orchestration were to resolve Namespace issues, data ambiguity, and data transformation. These data specific issues make workflows overwhelmingly large, complicated, and difficult to manage and maintain.

The various workflow specifications mentioned earlier extensively use the Extensible Markup Language (XML) to describe the orchestration script. The use of XML standardized the scripting language for platform independence but it also over-complicates even the simple tasks. For instance the simplest assignment activity in the BPEL4WS script for a single element in a complex type is shown below:

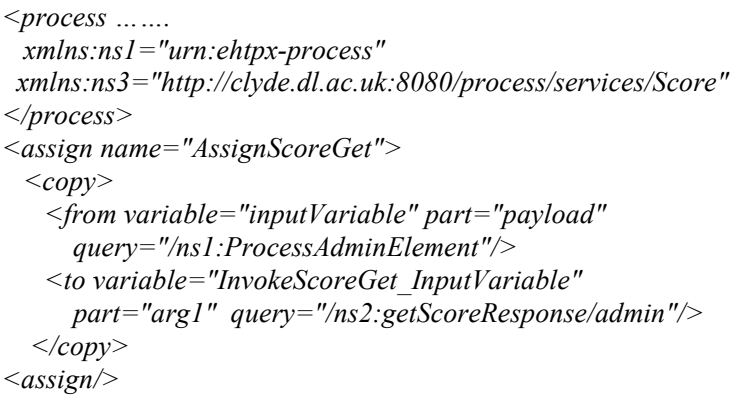

These data issues become increasingly more complicated when it comes to different developing styles for the Web Services. Loosely coupled Web Services (with data and corresponding Web Services loosely bound) use generic data types like "String" even for the complex data types which make any type of data validation impractical.

Namespace issues are more obvious when integrating RPC/encoded and Document/literal style Web Services. The RPC/encoded Web Services have limited support for Namespaces and different Namespaces cannot be mixed in complex data types. On the contrary, Document/literal style Web Services have more Namespace support and are compliant to WS-Interoperability Basic Profile [14].

With loosely coupled services, the orchestration engine plays a vital role and is involved in each activity directly or indirectly. Every message passed between different adjacent services has to pass through the workflow engine, which may solve data mapping issues prior to calling the next service.

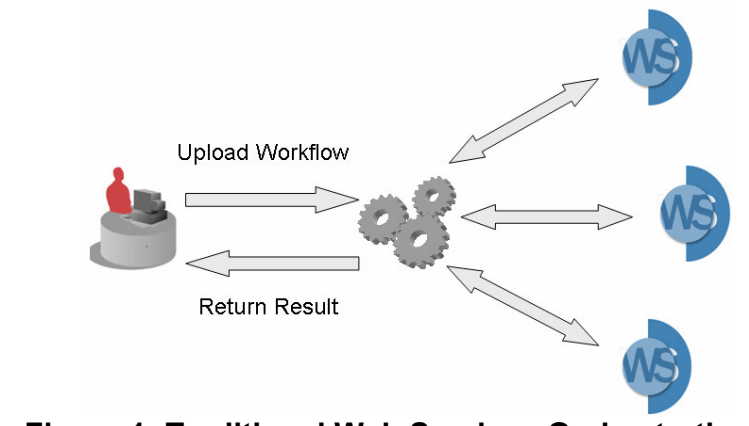

Figure 1: Traditional Web Services Orchestration

Current Web service descriptions allow operations to define the direction of messages (incoming or outgoing), but they do not describe the behaviour of the service involving multiple individual operations - in other words, they do not provide enough details of what the service can be expected to do. Such ambiguity 
requires extra alterations and assumptions when integrating, resulting in fragile workflows. Web Services compliant to any pre-defined data models give a better view of their behaviour, functionality and context in which they can be used.

Today's service descriptions may be adequate for simple information retrieval in a stateless message exchange, such as a stock quote. There is no standard way, however, of correlating the messages that are exchanged by the Web service with the context of the process in which the Web service operates. This is an important function for building useful service collaboration, and this is the area that Data-Centric workflow addresses.

\section{Web Services Resource Framework}

Web Services lack the notion of state, stateful interactions, resource lifecycle management, notification of state changes, and support for sharing and coordinated use of diverse resources in dynamic 'virtual organizations' [6]: issues that are of central concern to the developers of distributed systems. To address these problems, two important sets of specifications: WS-Resource Framework and WSNotification [23], built on the broadly adopted Web Services Architecture [15] and compliant with the WSInteroperability Basic Profile, were proposed.

WS-RF originates from the Grid paradigm which can be described as "coordinated resource sharing and problem solving in dynamic, multi-institutional virtual organizations". WS-RF specifications are based on the Extensible Markup Language (XML) schemas, and Web Services Definition Language (WSDL) interfaces for the properties and ports common to all WS-RF resources. WS-RF comprises four inter-related specifications; which define how to represent, access, manage, and group WS-Resources:

- WS-ResourceProperties [16] defines how WSResources are described by XML documents that can be queried and modified;

- WS-ResourceLifetime [17] defines mechanisms for destroying WS-Resources;

- WS-ServiceGroup [19] describes how collections of Web Services can be represented and managed;

- WS-BaseFaults [18] defines a standard exception reporting format.

\subsection{WS-Resources}

WS-Resources model the state of a Web Service by wrapping atomic/composite data types called WSResource Properties. A Resource Property is a piece of information defined as part of the state model, reflecting a part of the WS-Resource's state, such as its meta-data, manageability information and lifetime.

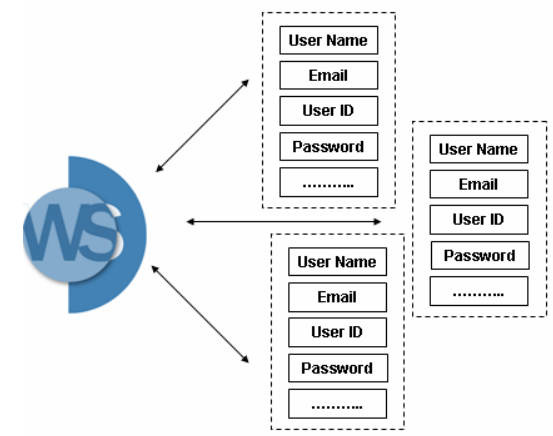

Figure 2: A WS-Resource with Resource Properties

The WS-RF specification supports dynamic insertion and deletion of the Resource Properties of a WS-Resource at run time. WS-Resource itself is a distributed object, expressed as an association of an XML document with a defined type attached with the Web Service portType in the WSDL. Although WSResource itself is not attached to any Uniform Resource Locator (URL), it does provide the URL of the Web Service that manages it. The unique identity of the WS-Resource and the URL of the managing Web Service is called an Endpoint Reference (EPR), which adheres to Web Services Addressing (WSA) [20]. WSRF avoids the need to describe the identifier explicitly in the WSDL description by instead encapsulating the identifier within its EPR and implicitly including it in all messages addressed through it.

WS-Resources instances have a certain lifetime which can be renewed before they expire; they can also be destroyed pre-maturely as required by the application.

\section{WS-Resource and Workflow}

Each workflow can be a stateful Web Service with its own Data Model encapsulated as a WS-Resource. The flexibility of the WS-Resource specifications and its reliance on the XML Schema provides natural way to capture all data information required to model the workflow. Intermediate data can be inserted or deleted from the WS-Resource during the life cycle of the workflow for monitoring and management purposes. WS-Resources can be used in different possible ways in the workflow without extending the specifications. The following section discusses different possible uses of WS-Resources in our Data Centric Workflow: 


\subsection{WS-Resource Sharing}

WS-Resources are not bound to a single Web Service; in fact multiple Web Services can manage and monitor the same WS-Resource instance with different business logic and from a different perspective. Similarly, WS-Resources are not confined to a single organization and multiple organizations may work together on the same WS-Resource leading to the concept of collaboration. Passing a unique identity of the WS-Resource instance between partner processes and organizations results in minimum network overhead and avoids issues of stale information. The WS-Resource EPRs are generated dynamically and can be discovered, inspected and monitored dynamically via dedicated Web Services. In Figure 3, Resource B is shared between two different Web Services each of them exposing possibly different sets of operations on the same WS-Resource, related to its role in the whole workflow orchestration.

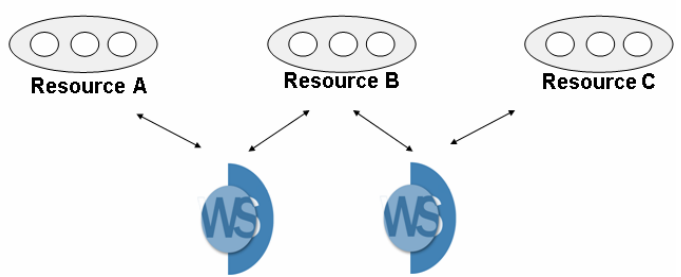

Figure 3: Web Service managing multiple WSResources and sharing same WS-Resource.

WS-Resource sharing is very effective for Data Centric workflows as different partner services can work on the single WS-Resource in the standard way. Web Services share the same view of the data structure with the single meaning. This minimizes the data specific issues in the Web Services orchestration. Changes in the single WS-Resources are applicable to all partner services.

\subsection{Managing Multiple WS-Resources}

In EAs, WS-Resources related to different entities can be very similar. Seller and Buyer details, for instance, are different WS-Resources in the Trading Application, but the majority of operations executed on these WS-Resources are either queries or minor updates. It is more effective to manage these similarly natured operations on different WS-Resources with the single Web Service. In Figure 2, different instances of the same WS-Resource are being managed by a single Web Service; whereas in Figure 3, multiple Web Services are managing different WS-Resources which can have any number of instances.
A single Web Service managing multiple WSResources could have its own inner mapping for different data models from different domains to achieve compatibility. WS-RF specifications support querying whole WS-Resources and even the specific parts of the WS-Resource through XPath or XQuery. Partner Web Services can query and retrieve the sub-set of the data model appropriate to their role and functionalities and commit the final changes. Single Web Service can be a part of different workflows sharing different WSResources and data models. It can be even used multiple times during different stages of a single workflow, working on different data set of the same data model (although similar in nature).

\subsection{WS-Resource Referencing}

WS-Resources are composed of Resource Properties which reflect their state. These can vary from simple to complex data types and even reference other WS-Resources. Referencing other WS-Resources through Resource Properties is a powerful concept which defines inter-dependency of the WS-Resources at a lower level. This eliminates complicated business logic in a similar way to the mapping of Entity Relationships in a Relational Database through primary and foreign keys. In EAs entities do not exist in isolation but inter-communicate and are dependent on each other's state. Similarly, WS-Resources are not only dependent on the state of other WS-Resources but can even query and modify them. In a typical Trading System (see Figure 4), a single User may reference multiple Orders placed by that User on different occasions and each Order references varying numbers of items purchased as a part of a single Order.

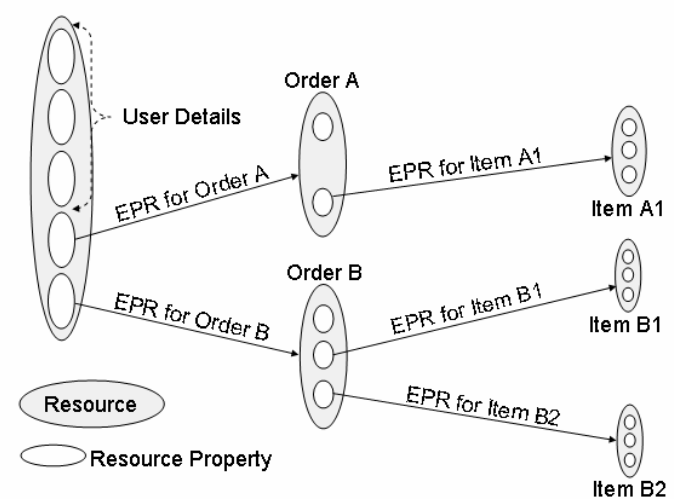

Figure 4: WS-Resources referencing other WSResources through their EPRs

In the data centric workflow context, WS-Resource referencing can provide extreme flexibility while 
designing the Data Model for the whole workflow. The flexibility in the workflow is one of the key requirements for the effective workflow as discussed in section 2. Optional data (independent of the working of the workflow) can be injected in the Data Model through references rather than changing the Data Model itself. For instance the monitoring, management or the log information can be coupled with the Data Model for recovery, steering and analysis purposes. Even the optional data coupled with the Data Model is queried based on the standard such as EPR and WS-RF Query Operations and thus never creates data related issues.

\section{Proof of Concept Implementation}

We have implemented the prototype of our sample application as stateful Web Services by modelling the data before implementation. The workflow implementation was a simple integration problem even though it involved the realisation of several workflow patterns [21].

The WS-RF specifications recommend the use of the Implied Resource pattern (Figure 5) to describe views on state and to support its management through associated properties. The Implied Resource pattern has a single Factory Service to instantiates the resources and an Instance Service to access and manipulate the information contained in the resources according to the business logic.

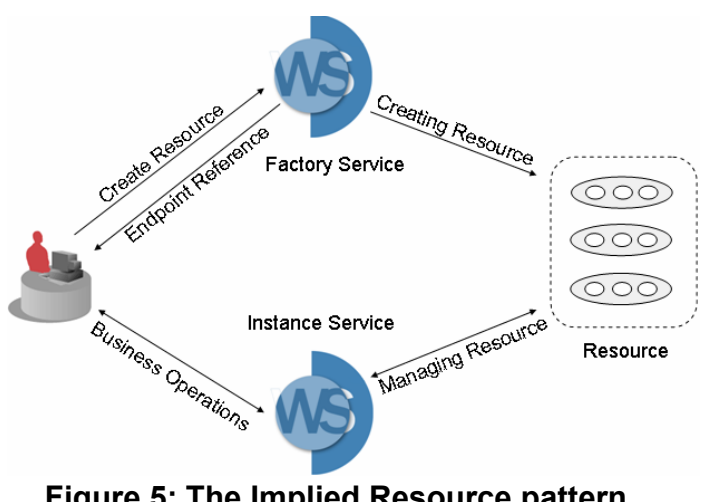

Figure 5: The Implied Resource pattern

The Data Centric workflow was developed as a stateful Web Service and its data model was encapsulated as a WS-Resource. The instantiation of the workflow was done through its Factory service; which also instantiate the main WS-Resource. The factory service also instantiates partner services through their corresponding factory services and maintains the list of EPRs of partner services. Each partner service is also a WS-RF based service and can have its own WS-Resource which may be outside the workflow domains. The Instance service for the workflow provides two sets of methods: methods for instantiating the workflow and those for monitoring, managing and querying the instantiated workflow. The Instance services related to partners expose different operations according to their roles and functions in the workflow context. Each operation takes the EPR of their main WS-Resource (encapsulating the data model of the workflow). This minimizes the requirement to pass data between the partner services which could have network overhead.

As expected the most time consuming activity was the modelling of the data related to the workflow. The application specific data was modelled separately in various XML Schema files using different Namespaces. To simulate the real world problem, different Namespaces were mixed in the complex data types by using <xsd:import $>$ and $<x s d$ include $>$. Separation of the data from the Web Services forced us to use Document/literal style Web Services, as recommended by WS-Interoperability Basic Profile.

The development of the individual Web Services was straightforward even though each service had Factory and Instance services according to the Implied Resource Pattern. The business logic of the workflow was implemented in the Instance service of the workflow which called the partner services according to the pre-defined sequence. The prototype development revealed that:

1. Minor changes in the Data Model and in the partner services don't require changes in the main instance service of the workflow;

2. Minor changes in the Data Model (restructuring of the data) had no impact on the workflow;

3. Severe changes in the Data Model may require changes in the partner services;

4. Interface changes of the partner services require changes in the workflow;

5. WSDL for the partner services was easy to manage and update;

6. Modelling data before implementation solves issues related to the automatic WSDL generation from various tools i.e. JAVA2WSDL for Axis, wscompile for JAX RPC and wsdl.exe for .Net platform;

7. Workflow calls partner services in predefined sequence without any complicated data mapping and transformation logic;

8. Partner services can even call the next service in the sequence without involving the main workflow service. 


\section{Conclusion}

The earlier hype of Web Services failed to deliver due to its initial coupling with the RPC style, as apparent in the tools provided by vendors of SOAP engine implementations. Similarly the existing culture of scripting workflows based on the operations of available services is impractical. Several composition languages for Web Services have emerged in recent years but all use a similar approach [22], which too has failed to meet the developers' and users' expectations. Most of the developers avoid direct interaction with $\mathrm{XML}$, resulting in the need for tools that automatically generate the WSDL. These tools complicate the situation due to their varying level of compliance and support for the standards. Even the WSDL generated from different versions of the same tool may not be compatible [24]. These shortcomings are more obvious in workflow scripts when dealing with data type mapping and transformation. We believe that starting with the Data Modelling and taking a top down (WSDL first) approach is required to ensure a consistent solution for orchestration. Defining a common platform-independent data type system facilitates the separation of roles, whereby workflow can be developed in isolation from partner services. In our experience, this greatly increases productivity and simplifies development, especially for large distributed applications where developers may be geographically separated.

\section{References}

[1] Gunter Kickinger, Peter Brezany, and Ivan Janciak, "User Driven Workflows for the Collaboration of Grid Services", MIPRO 2004, Opatija, Croatia, May, 2004.

[2] Sriram Krishnan, Patrick Wagstrom and Gregor von Laszewski, "A Workflow Framework for Grid Services", Technical report, Argonne National Laboratory, July 2002.

[3] Web Services Description Language (WSDL) 1.1, Available at http://www.w3.org/TR/wsdl.

[4] Peltz C, "Web Services orchestration and choreography" IEEE Computer 36(10), 46-52, 2003

[5] Globus Alliance, Web Service Resource Framework, Available at http://www.globus.org/wsrf/

[6] I. Foster, C. Kesselman, J. M. Nick, and S. Tuecke. The Physiology of the Grid: An Open Grid Services Architecture for Distributed Systems Integration, Globus Project, 2002.

[7] Peter J. Kammer, Gregory Alan Bolcer, Richard N. Taylor, Mark Bergnam, "Techniques for Supporting
Dynamic and Adaptive Workflow" Supported Cooperative Work (CSCW).

Computer

[8] Lucian-Mircea Patcas, John Murphy and Gabriel MiroMuntean, "Middleware Support for Data-flow Distribution in Web Services Composition", ECOOP Doctoral Workshop July 2005.

[9] Web Services Flow Language (WSFL) Version 1.0, http://www-06.ibm.com/software/solutions/webservices/ pdf/WSFL.pdf

[10] XLANG, available at http://www.gotdotnet.com/team/xml_wsspecs/xlangc/default.htm

[11] Business Process Execution Language for Web Services version 1.1 , ftp://www6.software.ibm.com/software/developer/librar y/ws-bpel.pdf

[12] Web Services Choreography Description Language Version 1.0, http://www.w3.org/TR/2004/WD-ws-cdl10-20040427/

[13] GSFL: An OGSA Workflow Framework, http://wwwunix.globus.org/cog/papers/gsfl-paper.pdf.

[14] Keith Ballinger, David Ehnebuske, Martin Gudgin, Mark Nottingham,Prasad Yendluri, Available at http://www.ws-i.org/Profiles/BasicProfile-1.0-2004-0416.html.

[15] D. Booth, H. Haas, F. McCabe, and et al. Web Services Architecture, W3C Working Group Note 11. Available at http://www.w3.org/TR/ws-arch/, 2004.

[16] Web Services Resource Properties 1.2 (WSResourceProperties), http://docs.oasis-open.org/wsrf /2004/06/wsrf-WS-ResourceProperties-1.2-draft-04.pdf.

[17] Web Services Resource Lifetime 1.2 (WSResourceLifetime), http://docs.oasis-open.org/wsrf/ 2004/06/wsrf-WS-ResourceLifetime-1.2-draft-03.pdf.

[18] Web Services Base Faults 1.2 (WS-BaseFaults), http://docs.oasis-open.org/wsrf/2004/06/wsrf-WSBaseFaults-1.2-draft-02.pdf.

[19] Web Services Service Group 1.2 (WS-ServiceGroup), http://docs.oasis-open.org/wsrf/2004/06/wsrf-WSServiceGroup-1.2-draft-02.pdf.

[20] D. Box, E. Christensen, F. Curbera, and et al. Web Services Addressing, W3C Member Submission 10 August 2004, Available at http://www.w3.org/Submission/ws-addressing, 2004.

[21] D. Hollingsworth, Workflow Management Coalition The Workflow Reference Model, 1995, http://www.wfmc.org/standards/docs/tc003v11.pdf.

[22] Van der Aalst, W.M., Dumas, M., ter Hofstede, A.H.: Web service composition languages: Old wine in new bottles? In: Euromicro Conference, 2003. Proceedings. 29th. (2003) $298-305$

[23] S. Graham, D. Hull, B. Muray, and et al. Web Services Notification, Version 1.3. Available at http://www.oasis-open.org/committees/wsn, 2005.

[24] A Akram, D Maredith and R Allan, "Best Practices in Web Service Style, Data Binding and Validation for use in Data-Centric Scientific Applications", e-Science All Hands Meeting, 2006 